\title{
Choice of a Metric for the Non-Hermitian Oscillator
}

\author{
D.P. Musumbu, H.B. Geyer and W.D. Heiss \\ Institute of Theoretical Physics and Department of Physics, \\ University of Stellenbosch, 7602 Matieland, South Africa
}

(Dated: June 14, 2021)

\begin{abstract}
The harmonic oscillator Hamiltonian, when augmented by a non-Hermitian $\mathcal{P} \mathcal{T}$-symmetric part, can be transformed into a Hermitian Hamiltonian. This is achieved by introducing a metric which, in general, renders other observables such as the usual momentum or position as non-Hermitian operators. The metric depends on one real parameter, the full range of which is investigated. The explicit functional dependence of the metric and each associated Hamiltonian is given. A specific choice of this parameter determines a specific combination of position and momentum as being an observable; this can be in particular either standard position or momentum, but not both simultaneously. Singularities of the metric are explored and their removability is investigated. The physical significance of these findings is discussed
\end{abstract}

PACS numbers: 03.65.-w, 03.65.Ge, 03.65.Ta 
There is continuing interest in the study of non-Hermitian Hamilton operators. Apart from the obvious situations relating to open systems, interest is focused upon a specific class of non-Hermitian operators giving rise to a real spectrum [1, 2, 3]. (See Ref.[4] for a recent survey and additional references.)

In Ref. [1] reality of the spectrum within the context of a consistent quantum mechanical framework is quite generally linked to the existence of a positive definite metric operator, giving rise to what is termed quasi-hermiticity, while in Ref. [2] it is conjectured from numerical evidence that for the class of non-Hermitian Hamiltonians studied there, reality of the spectrum results from symmetry under simultaneous parity and time reversal operations (denoted by $\mathcal{P}$ and $\mathcal{T}$ ) - so-called $\mathcal{P} \mathcal{T}$-symmetry. It has subsequently been strictly proven for particular $\mathcal{P} \mathcal{T}$-symmetry cases that the full spectrum is in fact real [5, 6]. (In Refs. [3] the relationship between quasi-Hermiticity [1] and $\mathcal{P} \mathcal{T}$-symmetry [2] is explored and elucidated in the context of pseudo-Hermiticity.)

One particular paradigm falling into this class is the simple non-Hermitian harmonic oscillator given by the Hamiltonian

$$
H=\omega\left(a^{\dagger} a+\frac{1}{2}\right)+\alpha a^{2}+\beta a^{\dagger 2}
$$

which is manifestly non-Hermitian for $\alpha \neq \beta$, but $\mathcal{P} \mathcal{T}$-symmetric $(\omega, \alpha$ and $\beta$ are real parameters). Here we use the usual boson operators

$$
a=\sqrt{\frac{\omega}{2}} \hat{x}+\frac{i}{\sqrt{2 \omega}} \hat{p}
$$

and correspondingly for $a^{\dagger}$, with $\hat{x}$ and $\hat{p}$ being the usual (Hermitian) position and momentum operators, respectively.

An extensive study of the properties of (11) has been undertaken in Ref. [7]. While the quoted paper is rather implicit, it has been followed by more explicit investigations $[8,9,10,11]$. The emphasis in [8, 10, 11] lies on the non-uniqueness of the metric with respect to which the non-Hermitian Hamiltonian appears as a quasi-Hermitian operator, i.e. Hermitian with respect to a non-trivial metric and its associated inner product, viz.

$$
\Theta H=H^{\dagger} \Theta
$$

with $\Theta$ being a positive Hermitian operator defining the new scalar product by

$$
\langle\cdot \mid \cdot\rangle_{\Theta}:=\langle\cdot \mid \Theta \cdot\rangle
$$


where $\langle\cdot \mid \cdot\rangle$ is the usual scalar product, employing the $L^{2}$-metric being the identity. Obviously (3) guarantees that the non-Hermitian $H$ is Hermitian with respect to $\langle\cdot \mid \cdot\rangle_{\Theta}$. Moreover, using the positive square root of the metric operator $\Theta$, the non-Hermitian $H$ can be transformed into a Hermitian operator with respect to the $L^{2}$-metric by the similarity transformation

$$
h_{S}=S H S^{-1}
$$

with $S^{2}=\Theta, S$ being likewise positive Hermitian. The essential point addressed explicitly in the present note is the non-uniqueness of $S$ and $\Theta$. In fact, various forms have been given in $[8,9,10,11]$.

In the spirit of a rather general investigation [1] about non-Hermitian Hamiltonians and their associated metric operators we present in this note a complete analysis of the whole range of operators $S$ yielding Hermitian operators $h_{S}$ according to (4) using (1) for $H$. For the problem at hand, our major finding is a continuous set of operators $S$ depending on one real parameter. Our emphasis lies on the physical significance of the specific choice of the metric in that a particular value of the parameter yields, apart from $h_{S}$, a further Hermitian operator (with respect to the $L^{2}$-metric) being another observable. Such further observable can be either the position or momentum operator, but in general a combination thereof, such as for instance the occupation operator.

We recall that the spectrum of $H$ is given by $E_{n}=(n+1 / 2) \Omega$ with $\Omega=\sqrt{\omega^{2}-4 \alpha \beta}$; of course it must coincide with that of $h_{S}$.

Guided by specific forms given in [8, 9, 10, 11] we make a general ansatz for $S$, viz.

$$
S=\exp A, \quad A=\epsilon a^{\dagger} a+\eta a^{2}+\eta^{*} a^{\dagger 2}
$$

being a positive Hermitian operator as long as $\epsilon^{2}-4 \eta \eta^{*}>0$ (the asterisk denoting complex conjugation); for this to hold $\epsilon$ must be real.

Using the expressions

$$
\begin{aligned}
S a S^{-1} & =\left(\cosh \theta-\frac{\epsilon}{\theta} \sinh \theta\right) a-2 \frac{\eta^{*}}{\theta} \sinh \theta a^{\dagger} \\
S a^{\dagger} S^{-1} & =\left(\cosh \theta+\frac{\epsilon}{\theta} \sinh \theta\right) a^{\dagger}+2 \frac{\eta}{\theta} \sinh \theta a
\end{aligned}
$$

with $\theta=\sqrt{\epsilon^{2}-4|\eta|^{2}}$ we obtain

$$
h_{S}=S H S^{-1}=U(\epsilon, \eta)\left(a^{\dagger} a+\frac{1}{2}\right)+V(\epsilon, \eta) a^{2}+W(\epsilon, \eta) a^{\dagger 2}
$$


for some $U, V$ and $W$ being obtainable after some algebra; the three functions depend in fact also on $\omega, \alpha, \beta$. Below explicit expressions are given for $h_{S}$ for the whole available range of the parameter $\eta$.

We require $h_{S}$ to be Hermitian, i.e. $U$ must be real and $V=W^{*}$. This leads to

$$
\frac{\tanh 2 \theta}{\theta}=\frac{\alpha-\beta}{(\alpha+\beta) \epsilon-2 \omega \eta}
$$

and $\eta=\eta^{*}$. The transformation (86) invokes a corresponding inverse transformation for the position and momentum operators occurring in $H$ [12, 13]. After suitable rescaling they read

$$
\begin{aligned}
& x=S^{-1} \hat{x} S=\cosh \theta \hat{x}+\frac{i}{\omega} \frac{\epsilon-2 \eta}{\theta} \sinh \theta \hat{p} \\
& p=S^{-1} \hat{p} S=\cosh \theta \hat{p}-i \omega \frac{\epsilon+2 \eta}{\theta} \sinh \theta \hat{x} .
\end{aligned}
$$

While $x$ and $p$ are by construction quasi-Hermitian with respect to the metric $\Theta$, and hence observables, these expressions clearly show that it is not clear a priori whether $\hat{x}$ or $\hat{p}$, or a suitable combination of those, remain observables when viewed in conjunction with the original Hamiltonian (11). In fact, such property depends on the particular choice of the metric. In the following we use instead of $\eta$ the parameter $z=\epsilon /(2 \eta)$ with $z \in[-1,1]$.

The relation (9) covers the whole range of possible parameter values that determine the metric. For a given set of parameters prescribing $H$ (that is $\omega, \alpha$ and $\beta$ ) we obtain from (9) a relationship between $z$ and $\epsilon$. In other words, the only free parameter that determines the metric is $z$ while $\epsilon$ is determined by

$$
\epsilon=\frac{1}{2 \sqrt{1-z^{2}}} \operatorname{arctanh} \frac{(\alpha-\beta) \sqrt{1-z^{2}}}{\alpha+\beta-z \omega} .
$$

Using the substitutions (21011) and (12) slightly tedious but straightforward algebra leads to the Hermitian set of Hamiltonians

$$
h_{S(z)}=\frac{1}{2}\left(\mu(z) \hat{p}^{2}+\nu(z) \hat{x}^{2}\right)
$$

with

$$
\begin{aligned}
& \mu(z)=\frac{-z(\alpha+\beta)+\omega-(\alpha+\beta-z \omega) \sqrt{1-\frac{\left(1-z^{2}\right)(\alpha-\beta)^{2}}{(\alpha+\beta-z \omega)^{2}}}}{(1+z) \omega} \\
& \nu(z)=-\omega \frac{z(\alpha+\beta)-\omega-(\alpha+\beta-z \omega) \sqrt{1-\frac{\left(1-z^{2}\right)(\alpha-\beta)^{2}}{(\alpha+\beta-z \omega)^{2}}}}{1-z} .
\end{aligned}
$$


The similarity transformation (15) that gives rise to (13) from (18) is obtained in a similar vein

$$
\begin{aligned}
S(z) & =\left(\frac{\alpha+\beta-\omega z+(\alpha-\beta) \sqrt{1-z^{2}}}{\alpha+\beta-\omega z-(\alpha-\beta) \sqrt{1-z^{2}}}\right) \frac{1}{4 \sqrt{1-z^{2}}}\left(a^{\dagger} a+\frac{z}{2}\left(a^{2}+a^{\dagger 2}\right)\right) \\
& =\left(\frac{\alpha+\beta-\omega z+(\alpha-\beta) \sqrt{1-z^{2}}}{\alpha+\beta-\omega z-(\alpha-\beta) \sqrt{1-z^{2}}}\right)^{\frac{1}{8 \omega \sqrt{1-z^{2}}}\left(p^{2}(1-z)+\omega^{2} x^{2}(1+z)-\omega\right)} .
\end{aligned}
$$

Specific cases have been given in [8, 9, 10, 11]:

- (i) for $z=0$ yielding from (12) $\epsilon=1 / 4 \log (\alpha / \beta)$ and thus

$$
\Theta=S^{2}=\left(\frac{\alpha}{\beta}\right)^{\frac{\hat{N}}{2}}
$$

and

$$
h_{S(z=0)}=\frac{\omega-2 \sqrt{\alpha \beta}}{2 \omega} \hat{p}^{2}+\frac{\omega}{2}(\omega+2 \sqrt{\alpha \beta}) \hat{x}^{2},
$$

- (ii) for $z=1$ yielding $\epsilon=-(\alpha-\beta) /(2(\omega-\alpha-\beta))$ and thus

$$
\Theta=S^{2}=\exp \left(-\frac{\alpha-\beta}{\omega-\alpha-\beta} \omega \hat{x}^{2}\right)
$$

and

$$
h_{S(z=1)}=\frac{\omega-\alpha-\beta}{2 \omega} \hat{p}^{2}+\frac{\omega \Omega^{2}}{2(\omega-\alpha-\beta)} \hat{x}^{2}
$$

- (iii) for $z=-1$ yielding $\epsilon=(\alpha-\beta) /(2(\omega+\alpha+\beta))$ and thus

$$
\Theta=S^{2}=\exp \left(\frac{\alpha-\beta}{\omega+\alpha+\beta} \frac{\hat{p}^{2}}{\omega}\right)
$$

and

$$
h_{S(z=-1)}=\frac{\Omega^{2}}{2 \omega(\omega+\alpha+\beta)} \hat{p}^{2}+\frac{\omega(\omega+\alpha+\beta)}{2} \hat{x}^{2} .
$$

We have presented $h_{S}$, that is the hermitized forms of $H$, in (13) and their special forms in (17), (19) and (21) in terms of the traditional momentum and position operators to indicate that they have all the same spectrum; they are simply rescaled forms of each other. In fact, while this is obvious by inspection from (17), (19) and (21), the general form (13) obeys as well identically the relation $\mu \nu=\Omega^{2}=\omega^{2}-4 \alpha \beta$, as it should. However, according to (10) and (11) the metric associated with a particular choice of $z$ does not - using the $L^{2}$-metric - yield Hermitian position and momentum operators. It does though yield the Hermitian combination

$$
O=\omega^{2} x^{2}(1+z)+p^{2}(1-z)
$$


which is - as we conclude from (10) and (11) - identical to the manifestly $L^{2}$-Hermitian operator

$$
\hat{O}=\omega^{2} \hat{x}^{2}(1+z)+\hat{p}^{2}(1-z)
$$

Note that $O=\hat{O}$ is Hermitian with respect to both the $L^{2}$-metric, being the identity, and the most general metric $\Theta(z)$ (compare also the final example in Ref. [12]). Note further that $z=0$ implies $O \sim \hat{N}=a^{\dagger} a$, the number operator. In contrast, $z=1$ yields, according to (10) and (11), a metric for which $x$ is $L^{2}$-Hermitian but $p$ is not. In fact, $S$ and thus $\Theta$ is now a function of $\hat{x}$ only and we read from (10)

$$
x=S^{-1} \hat{x} S=\hat{x} .
$$

Mutatis mutandis $z=-1$ gives a non-Hermitian $x$ but the Hermitian momentum

$$
p=S^{-1} \hat{p} S=\hat{p} .
$$

These results nicely demonstrate the point made in [1], and recently elaborated in [14, 15], in that the metric can be made unique by choosing, or constructing, further operators as observables (i.e. operators being quasi-Hermitian with respect to the same metric) to form an irreducible set comprising the Hamiltonian. The examples discussed in detail specify one more operator to be chosen, that is (i) the number or (ii) the position or (iii) the momentum operator.

While the specific choices made for $z$ may be physically appealing as one of each choice allows at least one of the three operators $(\hat{N}, \hat{x}, \hat{p})$ to be an observable in conjunction with the non-Hermitian Hamiltonian (1), any other choice of $z \in[-1,1]$ may be possible in principle. Such other choice yields, however, another Hermitian combination of the momentum and position operator as given in (22). Whether such combination has any particular physical meaning had to be judged by the specific case considered.

In turn, depending on the choice of parameters for $H$ (while duly observing $\omega^{2} \geq 4 \alpha \beta$ ), there may be combinations that don't allow a real solution for $\epsilon$ of (12) even if $z$ is properly chosen in the interval $[-1,1]$. In fact, the obvious requirement that the argument of the hyperbolic arctanh is not greater than unity - which is equivalent to the square root occurring in (14) being real - reveals that there is no real solution for $z \in\left[z_{-}, z_{+}\right]$with

$$
z_{ \pm}=\frac{(\alpha+\beta) \omega \pm(\alpha-\beta) \Omega}{\omega^{2}+(\alpha-\beta)^{2}}
$$


The numerical example $\omega=1, \alpha=1 / 2, \beta=1 / 4$ yields $[0.54 \ldots, 0.87 \ldots]$ as the disallowed region for $z$. Note that $z_{+}=1$ for $\omega=\alpha+\beta$. This combination is obviously incompatible with the choice $z=1$ as seen from (18) and (19). In other words, for $\omega=\alpha+\beta(\alpha \neq \beta)$ the position operator simply cannot be Hermitian. We stress that as $h_{S}$ fails to be Hermitian when $z \in\left[z_{-}, z_{+}\right]$, the metric $S$ is ill defined for these values of $z$ as the argument to be exponentiated in (15) is negative. The metric is singular (infinity) at $z=z_{-}$and zero at $z=z_{+}$.

The singularity just described of the metric is spurious, however. It means that it is removable [11] by making another choice for the metric, yet at the expense of trading in singularities elsewhere. For the present problem this is achieved by simply making the replacement $z \rightarrow-z$ everywhere. This entails in particular that

- in (14) $\mu(z)$ is to be replaced by $\mu(-z), \nu(z)$ by $\nu(-z)$ and in (15) $S(z)$ by $S(-z)$

- the region where the metric is ill defined is now at $z \in\left[-z_{+},-z_{-}\right]$

- $x \equiv \hat{x}$ for $z=-1$ with $p$ non-Hermitian

- $p \equiv \hat{p}$ for $z=+1$ with $x$ non-Hermitian

- item(ii) leading to (18) and (19) must now read

- (ii) for $z=-1$

- with the expressions following remaining unchanged

- item(iii) leading to (20) and (21) must now read

- (iii) for $z=1$

- with the expressions following remaining unchanged

- (22) now reads $O=\omega^{2} x^{2}(1-z)+p^{2}(1+z)$ and correspondingly for $\hat{O}$.

It is worth mentioning that the singularities of the metric persist if the parameters of the Hamiltonian are chosen such that $z_{+}$and $z_{-}$coincide. Using (23) this happens when $\Omega=0$ - ignoring the trivial case $\alpha=\beta$-, that is at an exceptional point [16, 17, 18], where all energies coalesce. With $\omega=2 \sqrt{\alpha \beta}$ the expression reads for $S(z)$

$$
S(z)=\left(\frac{\alpha+\beta-2 \sqrt{\alpha \beta} z+(\alpha-\beta) \sqrt{1-z^{2}}}{\alpha+\beta-2 \sqrt{\alpha \beta} z-(\alpha-\beta) \sqrt{1-z^{2}}}\right)^{\frac{1}{8 \omega \sqrt{1-z^{2}}}\left(p^{2}(1-z)+\omega^{2} x^{2}(1+z)-\omega\right)} .
$$


When $z \rightarrow z_{+}$the denominator of (24) vanishes to second order. The metric is singular at the exceptional point, which in a more general situation would be indicative of a phase transition [11, 18, 19].

Having completely analysed the Hamiltonian considered there remains the question: what choice to make to obtain unique physical answers? In this context we stress that, while the hermitized Hamiltonians have the same spectrum, the corresponding wave functions do depend on $z$. In fact, the set of Hamiltonians (13) clearly yield the well known harmonic oscillator wave functions but with distinctly different arguments for the Gaussian and Hermite polynomials, the respective arguments being given by the combination $(\nu / \mu)^{1 / 4} x$. In other words, not only is the preferred choice of the additional observable a function of $z$ and thus of the metric, but so are other variables such as transition matrix elements [20]. We are not aware of a convincing argument that could fix the choice of the metric. There remains an ambiguity. Any further elucidation should come from a genuine physical situation that is described by a $\mathcal{P} \mathcal{T}$-symmetric or other non-Hermitian Hamiltonian which is quasi-Hermitian, with a real spectrum. If it exists, Nature will tell what metric she prefers under given circumstances.

Note added in proof: Using arguments based on a perturbative approach it has been suggested [21] that the classical limit of the hermitized Hamiltonian be independent of the particular choice of the metric. Our nonperturbative results do not support this suggestion. In fact, the quantities $\mu(z)$ and $\nu(z)$ in (14) explicitly depend on $z$ and so does the Hamiltonian in (13). While the oscillator frequency is of course independent of $z$, the mass term becomes $1 / \mu(z)$ and the classical energy $E_{\mathrm{cl}}=A^{2} \Omega^{2} /(2 \mu(z))=\nu(z) A^{2} / 2$ ( $A=$ amplitude

of the classical oscillation). The (spurious) singularities at $z_{ \pm}$given in (23) also appear in the mass term; the mass and classical energy remain finite at $z_{ \pm}$but they are complex for $z \in\left[z_{-}, z_{+}\right]$. We note that the metric operator has an essential singularity in the classical limit $(\hbar \rightarrow 0)$, that is it cannot be expanded in powers of $\hbar$.

\section{Acknowledgement}

We thank Frederik Scholtz for a critical reading of the manuscript and for numerous illuminating discussions.

[1] Scholtz F G, Geyer H B and Hahne F J W 1992, Ann. Phys. (N.Y.) 21374 
[2] Bender C M and Boettcher S 1998, Phys. Rev. Lett. 804243

[3] Mostafazadeh A 2002, J. Math. Phys. 43 205; Mostafazadeh A 2002, J. Math. Phys. 43 2814; Mostafazadeh A 2002, J. Math. Phys. 433944

[4] Geyer H B, Heiss W D and Znojil M (Eds.) 2006, J. Phys. A: Math. Gen 39, No. 32 (Special issue on The Physics of Non-Hermitian Operators)

[5] Dorey P, Dunning C and Tateo R 2001, J. Phys. A: Math Gen. 345679

[6] Shin K C 2002, Commun. Math. Phys. 229543

[7] Swanson M S 2004, J. Math. Phys. 45585

[8] Geyer H B, Snyman I and Scholtz F G 2004, Czech. J. Phys. 541069

[9] Jones H F 2005, J. Phys. A: Math. Gen. 381741

[10] Scholtz F G, Geyer H B 2006, Phys. Lett. B 63484

[11] Scholtz F G, Geyer H B 2006, J. Phys. A: Math. Gen. 3910189

[12] Kretschmer R and Szymanowski L 2004, Czech. J. Phys. 5471

[13] Mostafazadeh A and Batal J 2004, J. Phys. A: Math. Gen. 3711645

[14] Znojil M, Geyer H B 2006, Phys. Lett. B 64052

[15] Mostafazadeh A and Ozcelik S 2006, quant-ph/0607120

[16] Kato T 1976, Perturbation Theory for Linear Operators 2nd edn (Heidelberg: Springer)

[17] Heiss W D and Sannino A L 1990, J. Phys. A: Math. Gen. 231167

[18] Heiss W D 2004, Czech. J. Phys. 541091

[19] Heiss W D 2006, J. Phys. A: Math. Gen. 3910081

[20] Musumbu D P 2006, MSc thesis, University of Stellenbosch (unpublished)

[21] Mostafazadeh A 2006, J. Phys. A: Math. Gen. 3910171 